\title{
Study of the Design and Assembly of a High Harmonic Fast Wave Antenna for an LAPD
}

\author{
Chao Yu $\mathbb{D}$, ${ }^{1,2}$ Qingxi Yang $\left(\mathbb{D},{ }^{1}\right.$ Yuntao Song, ${ }^{1}$ Jiahao Li $\mathbb{D},{ }^{1,2}$ Hao Xu, ${ }^{1}$ Xiaokang Yang, ${ }^{3}$ \\ Michl Binderbauer, ${ }^{3}$ Jon Schroeder, ${ }^{3}$ Yuanxu Song, ${ }^{3}$ Richard Goulding, ${ }^{4}$ \\ Bart Van Compernolle, ${ }^{5}$ Troy Carter, ${ }^{5}$ Ning Li, ${ }^{1}$ Yongsheng Wang, ${ }^{1}$ and Wei Song ${ }^{1}$ \\ ${ }^{1}$ Institute of Plasma Physics, Hefei Institutes of Physical Science, Chinese Academy of Sciences, Hefei, China \\ ${ }^{2}$ University of Science and Technology of China, Hefei, China \\ ${ }^{3}$ TAE Technologies, Foothill Ranch, CA, USA \\ ${ }^{4}$ Oak Ridge National Laboratory, Oak Ridge, TN, USA \\ ${ }^{5}$ University of California, Los Angeles, CA, USA
}

Correspondence should be addressed to Qingxi Yang; yangqx@ipp.ac.cn

Received 30 November 2020; Accepted 14 March 2021; Published 7 April 2021

Academic Editor: Peter Ivanov

Copyright ( $\odot 2021$ Chao Yu et al. This is an open access article distributed under the Creative Commons Attribution License, which permits unrestricted use, distribution, and reproduction in any medium, provided the original work is properly cited.

\begin{abstract}
The simulation survey of TAE Technologies has demonstrated that high harmonic fast wave (HHFW) heating is a promising method for core electron heating of FRC plasma. This study mainly describes the HHFW antenna mechanical design and assembly on the basis of the results of electromagnetic simulations performed by Oak Ridge National Laboratory (ORNL), the available port dimensions, and antenna installation position of the LAPD. Compared to the original scheme, this antenna is also optimized in the design. It is found that the $E$ field distribution of optimized antenna becomes even, and the maximum electric field decreases by approximately $14 \%$. The current on the antenna box and FS is reduced after optimization, whereas the maximum $J$ density decreases from $53.3 \mathrm{kA}$ to $14.5 \mathrm{kA}$. The reflection performance of the port at $30 \mathrm{MHz}$ is also improved after the structural optimization; The $k_{/ /}$spectrum distribution is sharper at the monopole phase $(0,0,0,0)$ and dipole phase $(0, \pi, 0, \pi)$ and $(0,90$, $270,180)$ than other phases. The optimized antenna can obtain a maximum $\left|k_{/ /}\right|$spectrum, which peaks about $\left|k_{/ /}\right|=30 \mathrm{~m}^{-1}$ at the dipole phase $(0, \pi, 0, \pi)$. The analysis results and assembly strategy can provide useful reference and guidance for the study of HHFW antenna design and fabrication in LAPD or other magnetic confined fusion devices.
\end{abstract}

\section{Introduction}

Antennas can transform guided waves propagating along transmission lines into electromagnetic waves propagating in unbounded media, or vice versa. Radio communication, radio, television, radar, navigation, electronic countermeasure, remote sensing, radio astronomy, and other engineering systems all use electromagnetic waves to transmit information and all rely on the antenna to work [1-4]. In addition, antenna is an important method of heating plasma in magnetic confined fusion devices. For instance, ion cyclotron radiofrequency (ICRF) heating is an important method of heating in tokamaks, and the main equipment used is antenna.
Effectively heating the core electrons of field-reversed configuration (FRC) plasma is challenging due to its unique magnetic field profile. A recent survey by Tri Alpha Energy (TAE) Technologies indicates that high harmonic fast wave (HHFW) heating, which has been successfully adapted to high beta, over-dense spherical Tokamak plasmas, such as the National Spherical Torus Experiment (NSTX), for the experiments of core electron heating and off-axis current drive, can balance the conflict between good wave accessibility and efficient power damping of electrons $[5,6]$. This discovery motivates a project on the experimental study of HHFW antenna-plasma coupling and wave propagation on a reliable test bench, such as the large plasma device (LAPD) at the University of California-Los Angeles (UCLA), before 
the method of HHFW electron heating is employed on the C-2W, an advanced beam-drive field-reversed configuration (FRC) device recently built at TAE. Therefore, in the framework of the collaboration agreement between TAE and Institute of Plasma Physics, Chinese Academy of Sciences (ASIPP), ASIPP is in charge of the design and fabrication of a high-power capable, phased array (four strap) HHFW antenna for the radio frequency $(\mathrm{RF})$ project of TAE on the LAPD at UCLA.

Ion cyclotron range of frequencies (ICRF) antennas have been used as an efficient heating method in many tokamak devices, such as the Tokamak Fusion Test Reactor (TFTR), Joint European Torus (JET), Experimental Advanced Superconducting Tokamak (EAST), and ITER. These antennas can provide steady high-power input with high efficiency in tokamaks [7]. However, the radio frequency (RF) sheath effect has been found to be detrimental. The RF rectification sheath potential not only reduces the heating performance of the ICRF antenna through the acceleration of ions but also causes a local hot spot on the ICRF structure, which has been observed during the experiments hosted in tokamaks. The JET experiment has shown that the maximum temperature on the ICRF structure can reach $800^{\circ} \mathrm{C}$ due to the hotspot [8]. The edgeRF phenomenon can not only affect the plasma density but also cause bright spots and erode the Faraday shield (FS). The fluxes of ions are accelerated in the RF-driven sheaths near the antenna, causing parasitic impurity sputtering, arcs, and hotspots on the antenna surface [9]. The RF sheath effect arises and narrows the gap between the FS elements, thereby increasing the electric fields and accelerating ions to an appreciable fraction of the gap voltage, which is approximately $1 \mathrm{kV}$ per gap for the TFTR and JET antennas [10]. The RF sheath effect can also be amplified due to FS elements that are imperfectly aligned with the combined poloidal and toroidal magnetic fields according to the research from Princeton Plasma Physics Laboratory (PPPL) [11]. The HHFW power loss is normally researched through the scrape-off layer (SOL) and plasma wall interaction (PWI). Due to the high heating power of the ICRF system, edge loss can be due to erosion or impurity production [12]. Certain developments in the RF sheath, which causes the ICRF-edge interaction, have shown that theorization, modelling, and experiments can be performed together to progress in the understanding and control of the ICRF-edge interaction in several tokamaks [13]. For example, TOPICA code was developed to compute the antenna near field for the study of RF-PSI effects. Some 1D particle-in-cell simulation codes were developed to investigate plasma sheath structures around a high-voltage transmitting antenna in the inner magnetosphere, and the results were assessed by experiments [14]. A device was designed to determine the method for predicting the performance of the ICRF antenna under the loss of RF sheath [15]. An LAPD, which is a linear device, was designed to accommodate various experiments for basic plasma physics research. An LAPD was designed with the capability to diagnose and model the effect of RF rectification sheath potential and impurity production on an
ICRF antenna. Some experiments on an LAPD were proposed to investigate the sheaths caused by an actively powered RF antenna [16].

\section{Motivation and Design Requirements of the HHFW Antenna}

Conventional electron heating scenarios, such as electron cyclotron resonant heating and fast wave mode conversion heating, which are widely utilized in tokamaks or stellations, cannot be adapted in field-reversed configuration (FRC) plasmas due to the conflict between the wave penetration into core plasmas and the efficiency of power damping on electrons. Given that an increase in electron temperature can help reduce charge exchange losses and increase fast particles that slow down time, thereby enhancing the efficiency of neutral beam heating and current drive and improving FRC plasma confinement and performance, numerous efforts have been recently made at TAE to explore various microwave and RF heating scenarios, including upper-hybrid resonant waves, electron Bernstein waves, Whistler (helicon) waves, and HHFW heating. Among these scenarios, studies with the GENRAY-C ray-tracing code have demonstrated that HHFW may be a promising path for the core electron heating of C-2U-like FRC plasmas. Under the motivation of these promising simulation results, $\mathrm{HHFW}$ is designed for the LAPD.

Based on the current RF generator and LAPD ports for the HHFW antenna, the design requirements are as follows:

(1) The impedance of the transmission lien is $50 \Omega$

(2) The motion range is from $0 \mathrm{~mm}$ to $200 \mathrm{~mm}$, adapting to the experimental requirements

(3) Easy installation and maintenance are required

\section{Design of the HHFW Antenna}

3.1. Integration of the HHFW Antenna. The HHFW antenna is composed of four modules, and each module comprises an FS, a current strap, and a transmission line (TL). To form a seal with the LAPD device, one adaptor tube connected with one bellow is sealed with a port and a movable plate by an $\mathrm{O}$ ring. One limiter made of $\mathrm{BN}$ is fixed between two modules, and four modules are fixed into integration by bolts on the top and bottom of the FS inside of the vacuum vessel (VV) and the flanges of the movable base outside of the VV. The HHFW antenna can move along the radius in the range from $0 \mathrm{~mm}$ to $200 \mathrm{~mm}$ by means of one driving motor located at the rear of the antenna because this adapts to the plasma operation scenario and meets the requirements of easy installation and maintenance. The detailed structure is shown in Figure 1.

3.2. FS. Four independent modules of FS are integrated into one large component for the HHFW antenna, as displayed in Figure 2, to protect the straps from particle bombardment. Each FS consists of eight bars and one box that are made of the stainless-steel SS 316L. On the basis of the easy assembly 


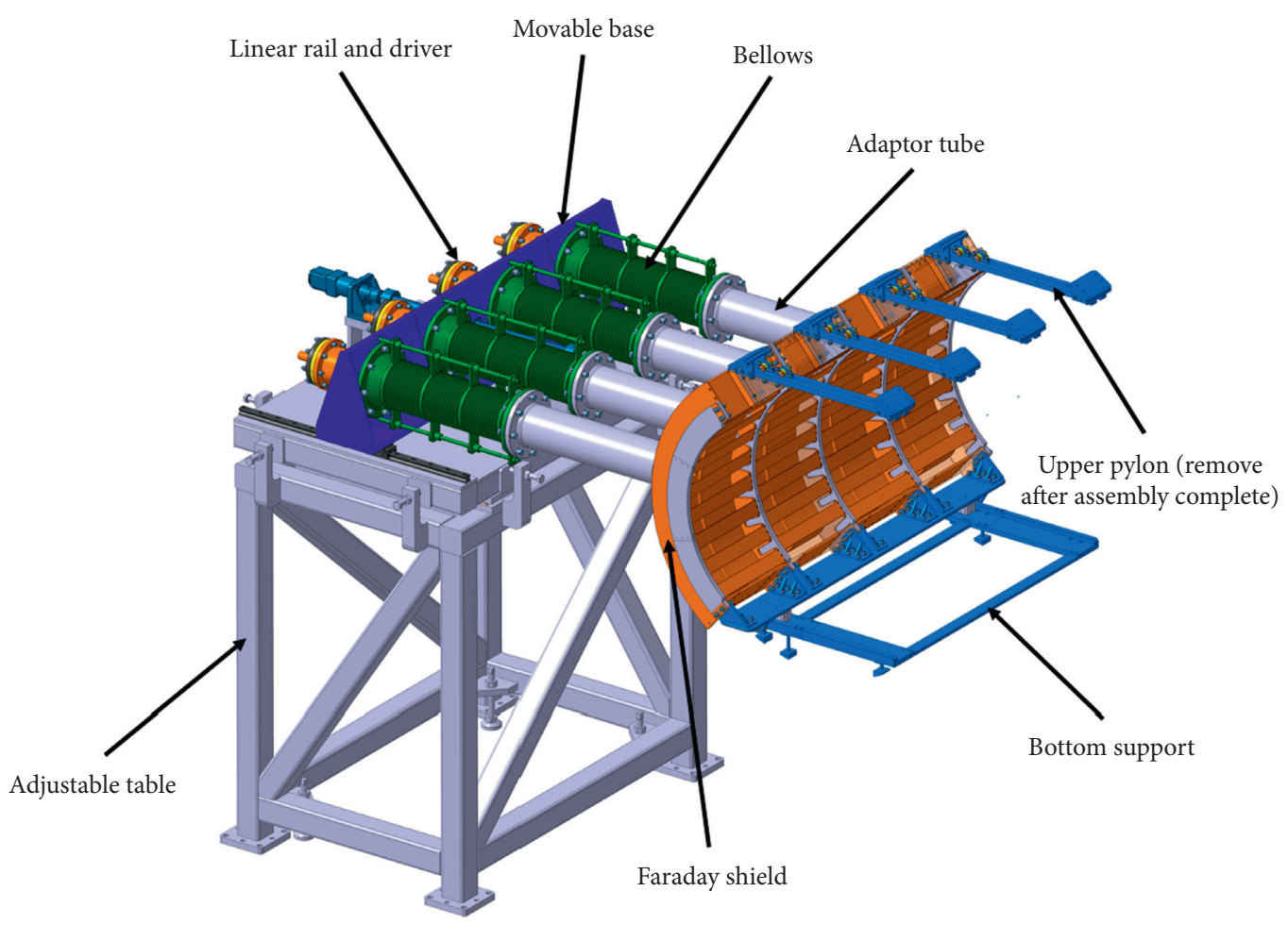

FIGURE 1: Brief sketch of the whole antenna system.

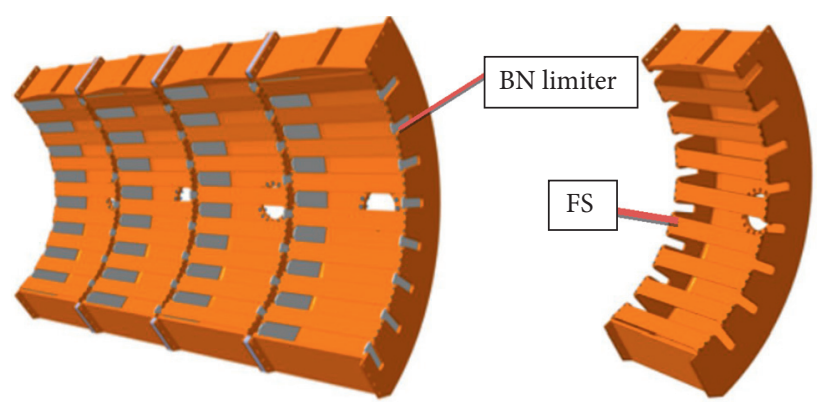

(a)

(b)

FIGURE 2: Integration of FS by four modules (a) and one module of FS (b).

and disassembly of the straps, the FS bars are fixed on the box by the bolts. Each box has many slots; thus, magnetic flux can penetrate the boundary. Two BN limiters are placed at both sides of each FS module to cover the slot to prevent connection between the plasma and the strap.

3.3. Strap and Transmission Line (TL). For the HHFW antenna, four straps are designed for the coupling power to plasma. Each strap is made of copper to reduce the RF loss, and one end is connected with the feed of an inner conductor, while the other is connected to the ground. The shape of the strap is designed as a curve to meet the plasma operation requirements.

The TL is composed of the inner conductor, outer conductor, and some supporting structures, which mainly transmit power from the generator to the plasma. For the HHFW antenna system, one end of the inner conductor serves as a feeder connected with a strap; the outer conductor is docked on the outer shell of the vacuum chamber, and the other is connected with the RF generator. Due to the $\mathrm{VV}$ port dimension limit, the front end of the TL is designed with a tapered shape, as shown in Figure 3.

In view of the requirement for a strap that is easy to assemble and limits deformation to within the requirements, the structure optimization of the strap and TL is conducted under gravity. Different thicknesses and materials are selected to perform the simulation. Based on the results, the strap thickness is $12 \mathrm{~mm}$ and the inner conductor thickness is $1.5 \mathrm{~mm}$. As illustrated in Figure 4, the deformation of the strap is $0.02 \mathrm{~mm}$, which is far below the design requirements.

3.4. Support System. To make the HHFW antenna stable and for safe operation, two trusses are designed to support the antenna: one is installed inside of the VV to support the 


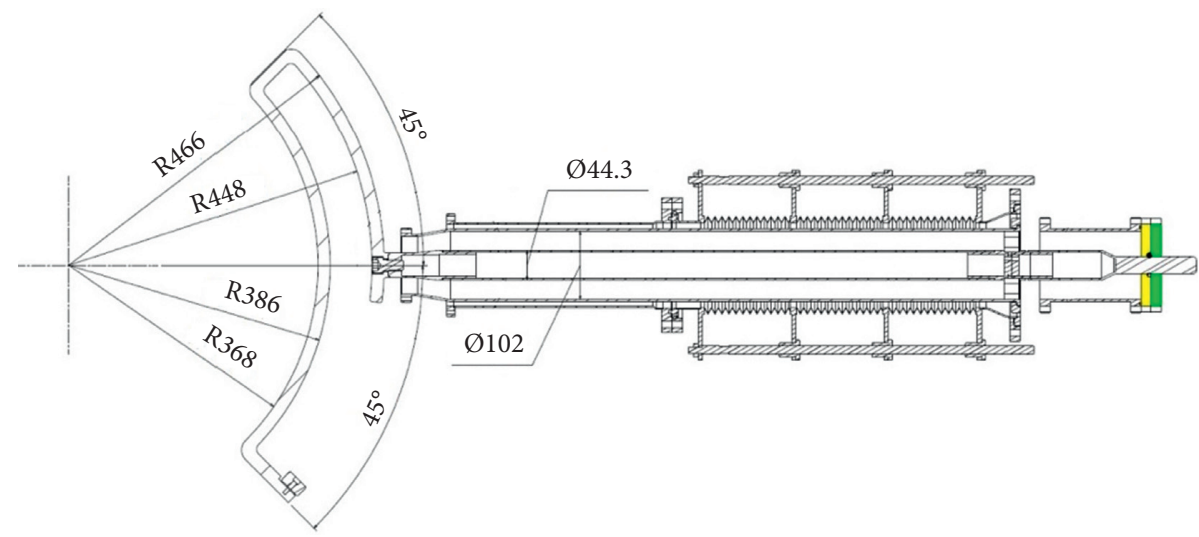

FIgURE 3: Detailed view of the strap connection with TL.
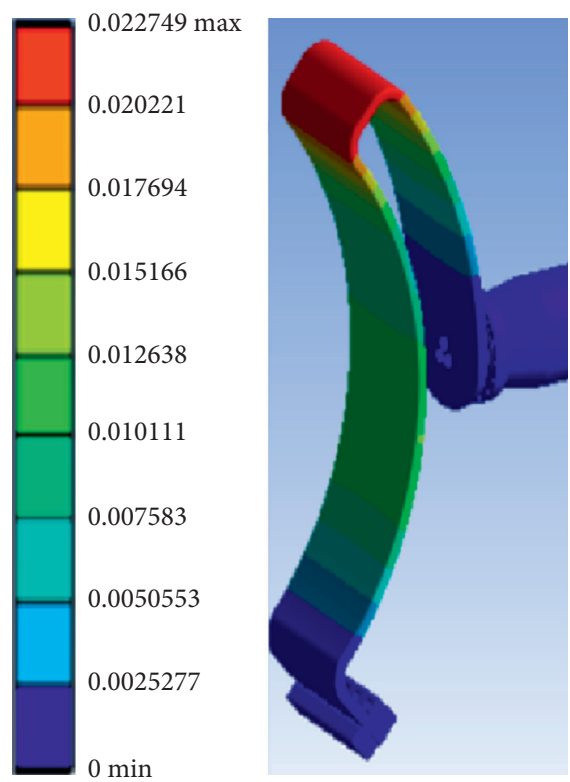

Figure 4: Deformation of the strap and TL of the HHFW antenna.

weight of the forepart of the HHFW antenna, and the other is fixed outside of the VV to support the rear part of the HHFW antenna. The detailed structure is shown in Figure 5.

To easily install four TLs, which are connected with the fore parts of the HHFW antenna through the VV ports, the rear truss is designed with an adjustment function so that it can be adjusted with three degrees of freedom in the $X_{-}^{-}, Y^{-}$, and Z-directions. Additionally, the fore truss has an adjustment structure to keep the top surface parallel with that of the rear truss within the design requirements, ensuring that the antenna can be moved smoothly along the radian direction in the range of $0 \mathrm{~mm}$ to $200 \mathrm{~mm}$. Apart from these trusses, four upper pylons are designed to temporarily support the rear parts of the HHFW antenna during installation and are removed after assembly.

3.5. Optimization of the Antenna Structure. In many tokamak experiments, hotspots are found when ICRF antennas work at a high-power feed; the impurities generated can then increase the RF loss and greatly affect the antenna performance, which is related to the high electric field on the antenna components [12]. With the help of the software HFSS, a simulation at $10-50 \mathrm{MHz}$ of the water load is conducted, and high electric field distribution and current densities are observed in some components of the structure. To reduce the possibility of generating impurities from high field and current densities on the antenna FS, straps, and box, two optimizations for the HHFW antenna are performed: one is the antenna box flipped at $180^{\circ}$, and the other has shifted FS elements. The original and optimized structures are presented in Figure 6.

Figure 7 shows the normalized electric field distribution of the two versions of the antenna at $30 \mathrm{MHz}$ and $(0,0,0,0)$, where the power fed into each port is $1 \mathrm{MW}$. The E field is normalized here according to the breakdown electric field limit given in [17]. The electric field value is relatively high in the upper area of the front end of the current straps before optimization, and it is almost in the same poloidal position. Figure 7 also displays that the overall electric field is still very high, even though the input power is relatively high; the field distribution becomes even, and the maximum electric field decreases by approximately 14\%. Figure 8 illustrates the normalized $J$ density distribution of the two versions of the antenna. The high current density area is mainly concentrated on the edge and at the end of the strap. The current on the antenna box and FS is reduced after optimization, whereas the maximum $J$ density decreases from $53.3 \mathrm{kA}$ to $14.5 \mathrm{kA}$ because the mutual coupling in the high field area is reduced to an extent due to the change in the location of adjacent straps with high fields. To minimize the fluctuation in the power spectrum change, we retain the strap structure but switch the feed and ground points. The shifted FS elements after optimization also intercept the flow of parallel currents, which contributes to the RF potential.

The S11 parameters before and after antenna optimization are also compared here, as shown in Figure 9. The reflection performance of the port at a certain frequency $(30 \mathrm{MHz}$ in this study) is improved after the structural optimization. For the optimized three-dimensional electromagnetic simulation model, the electromagnetic field distribution at about $3 \mathrm{~cm}$ in front of the antenna is taken for 


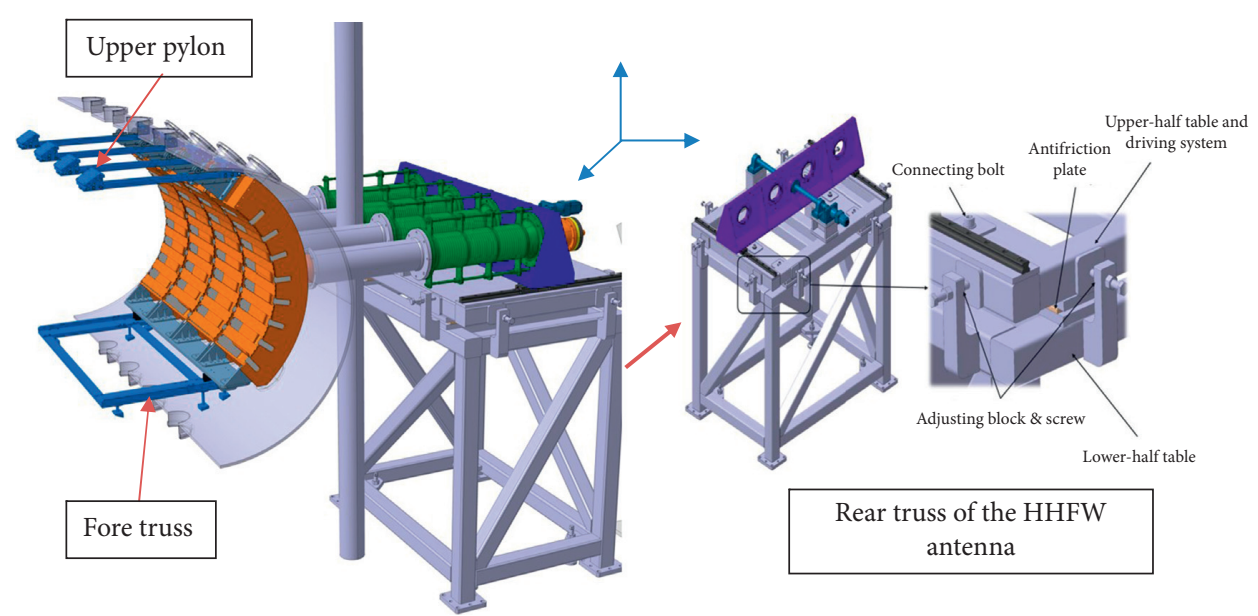

FIgURE 5: Structure of the HHFW antenna support.
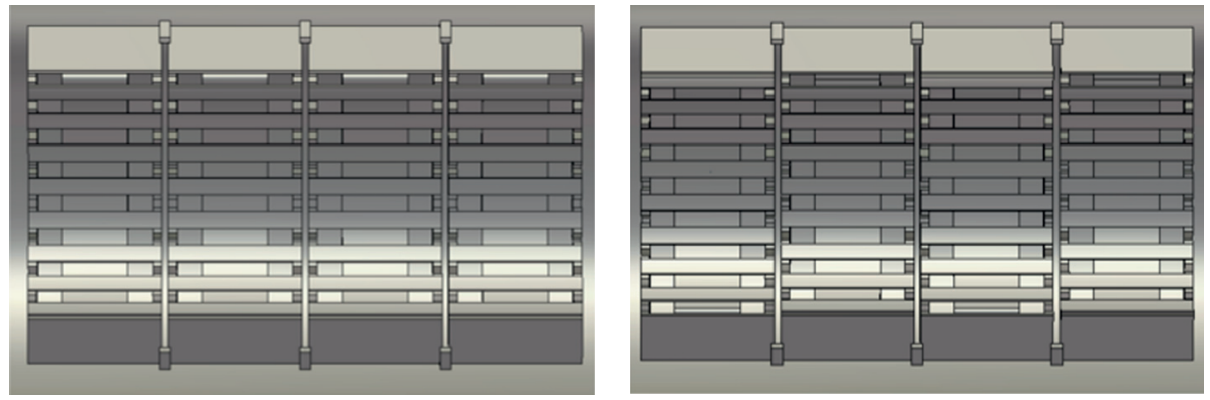

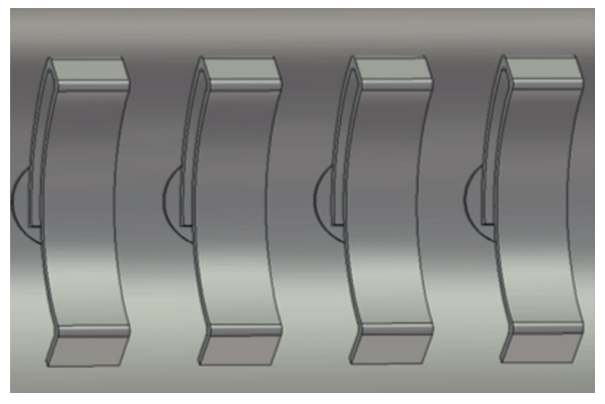

(a)

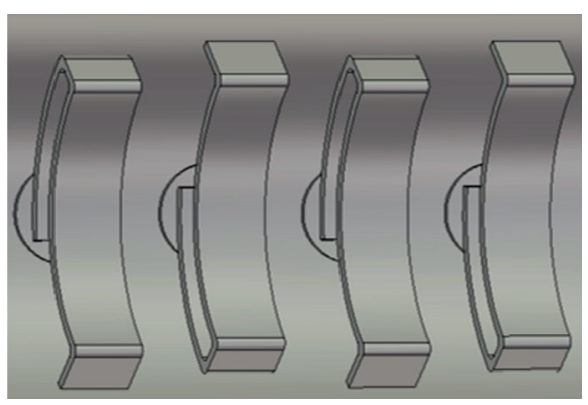

(b)

FIGURE 6: Original FS and strap array (a) and FS and strap array after optimization (b).
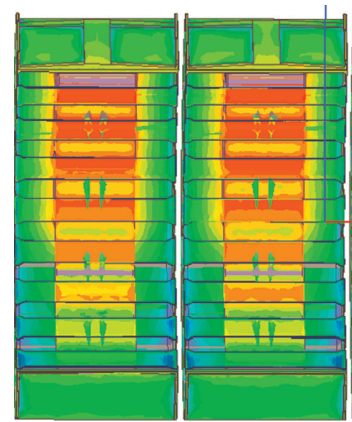

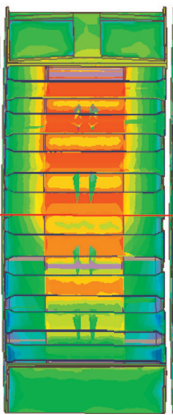

(a)
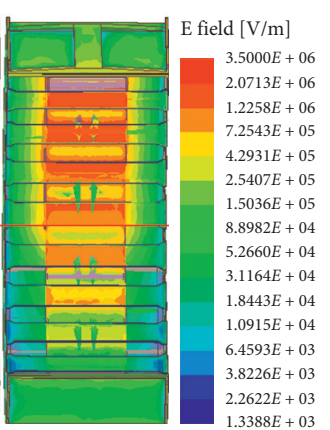

$1.3388 E+03$
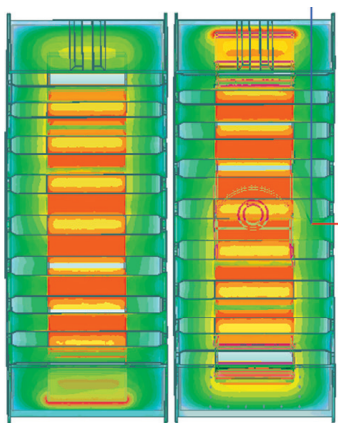
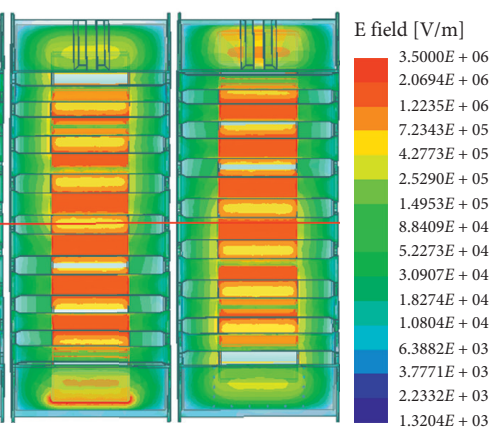

(b)

Figure 7: Normalized $E$ field distribution of the two versions of the antenna at $(0,0,0,0)$ and $30 \mathrm{MHz}$ before optimization (a) and after optimization (b). 


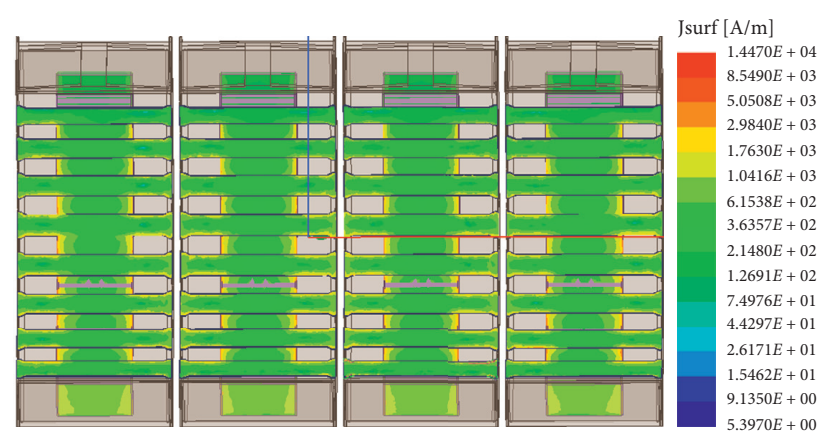

(a)
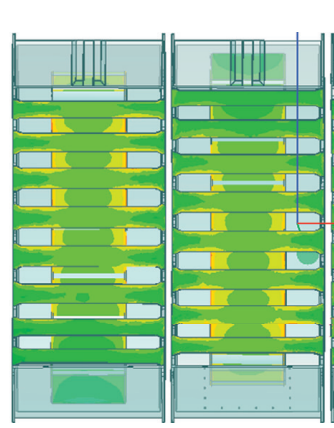

$\operatorname{Jsurf}[\mathrm{A} / \mathrm{m}]$
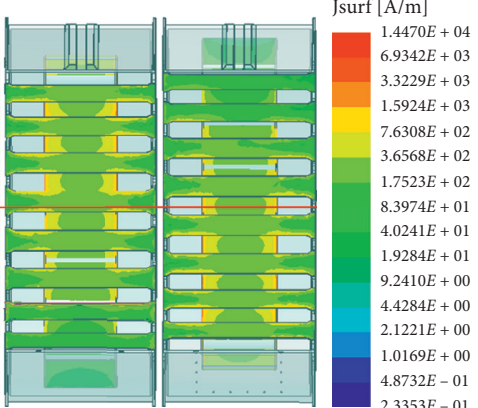

(b)

FIgURE 8: Normalized $J$ density distribution of the two versions of the antenna at $(0,0,0,0)$ and $30 \mathrm{MHz}$ before optimization (a) and after optimization (b).

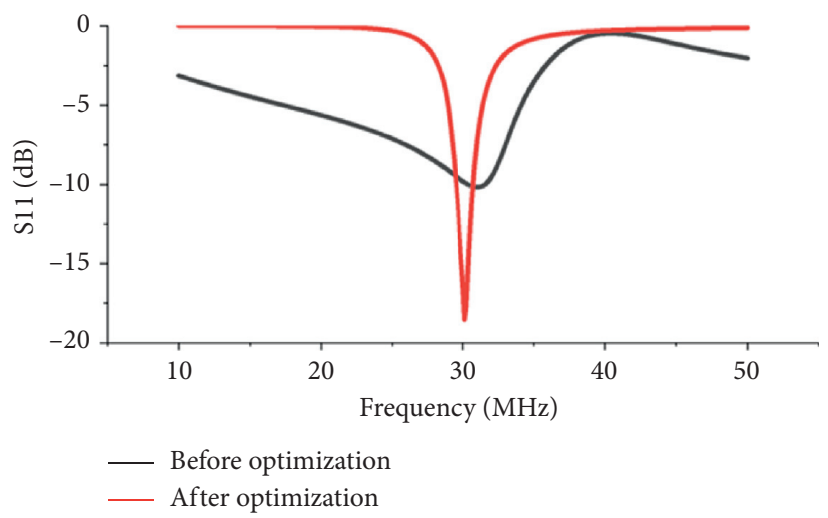

(a)

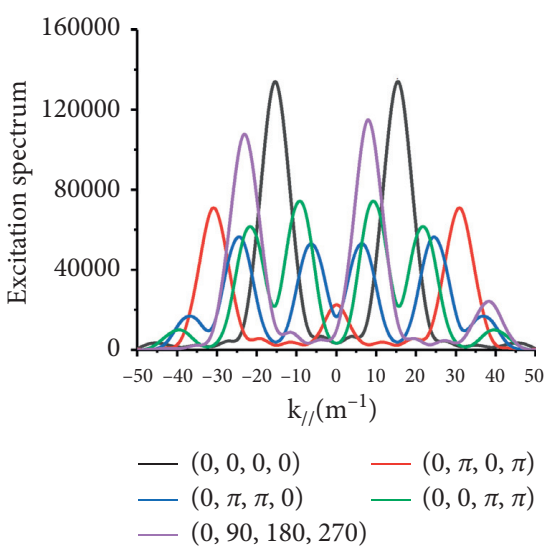

(b)

FIGURE 9: S11 comparison of the two versions of the antenna (a) and $k_{/ /}$spectrum of the optimized antenna (b).

$k_{/ /}$spectrum calculation. The results in Figure 9 show that the $k_{/ /}$spectrum distribution is sharper at the monopole phase $(0,0,0,0)$ and dipole phase $(0, \pi, 0, \pi)$ and $(0,90$, $270,180)$ than other phases, which is conducive to penetrating the cut-off layer of plasma boundary and has better coupling performance. The optimized antenna can obtain a maximum $\left|k_{/ /}\right|$spectrum, which peaks about $\left|k_{/ /}\right|=30 \mathrm{~m}^{-1}$ at the dipole phase $(0, \pi, 0, \pi)$.

\section{Assembly Strategy of the HHFW Antenna}

Considering that the HHFW antenna is integrated in the LAPD VV to participate in the plasma campaign, the assembly for the antenna not only is within design tolerances but also ensures that the antenna can move smoothly, adapting to different plasma configurations. To meet the assembly tolerances and to achieve simple installation, the assembly strategy for the HHFW antenna is investigated. On the basis of the HHFW antenna and VV structure, the fore truss in the $\mathrm{VV}$, four temporarily pylons, rear truss outside the VV, and outer conductors are installed using the measuring datum network and are placed according to the required position. Four FS modules, four inner conductors, and five ceramic baffles are sequentially installed in that order; the parallelism of the four outer conductors required is no more than $0.1 \mathrm{~mm}$. Subsequently, FS bars are installed on the FS boxes, and four small bottom plates and one large bottom base are connected to the FS. The temporary upper pylons are also removed, ensuring that the wheels are fixed on the bottom base for connection with the surface of the fore truss at the end. The assembly process and final assembly status of the HHFW antenna are shown in Figures 10 and 11, respectively. After the assembly is finished, the movable test of the antenna is conducted by the drive system. The result demonstrates that the antenna can be moved smoothly, and the stroke reaches $200 \mathrm{~mm}$, both of which satisfy the requirements.

\section{Summary}

In this study, we introduce the key components and dimensions of the HHFW antenna. Then, we compared the antenna performance before and after optimization. The simulation results have demonstrated that the $E$ field distribution of optimized antenna becomes even, and the maximum electric field decreases by approximately $14 \%$. The 


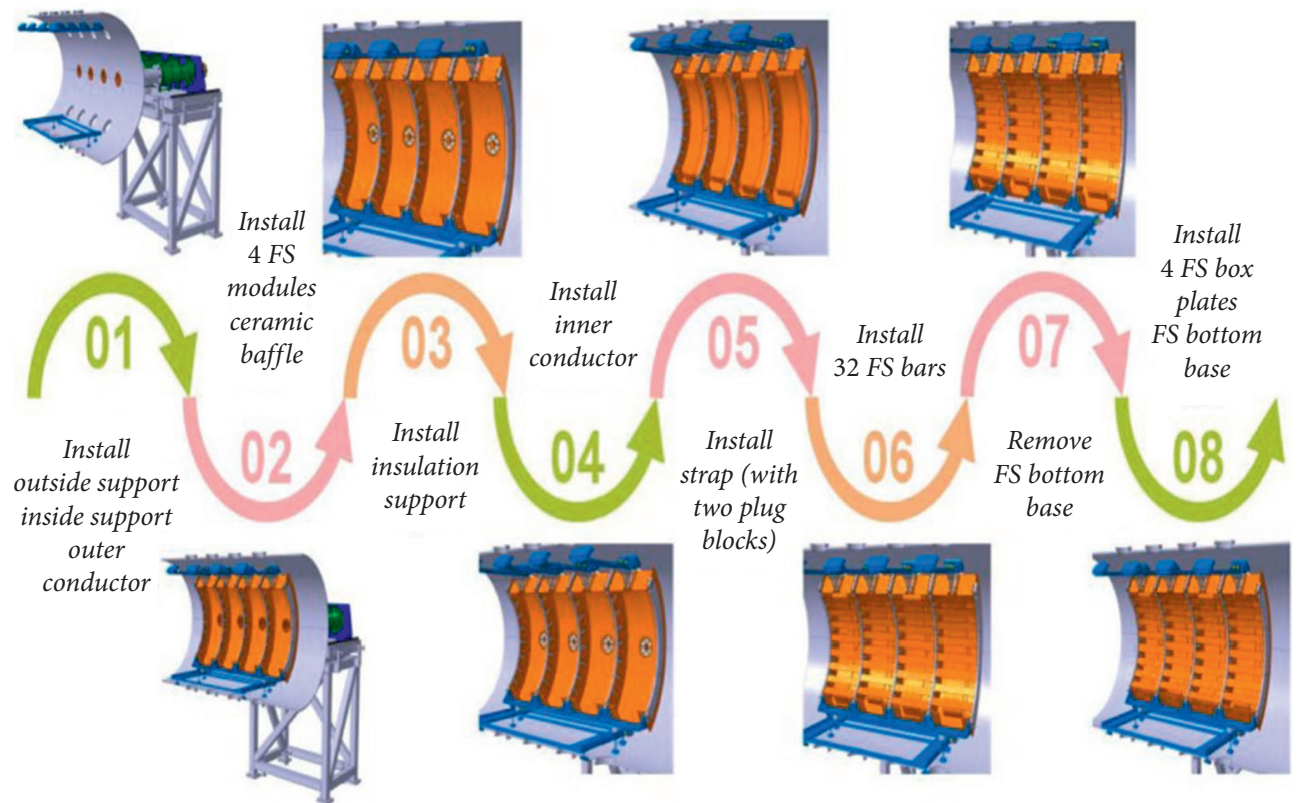

FIgURE 10: Assembly process of the HHFW antenna.

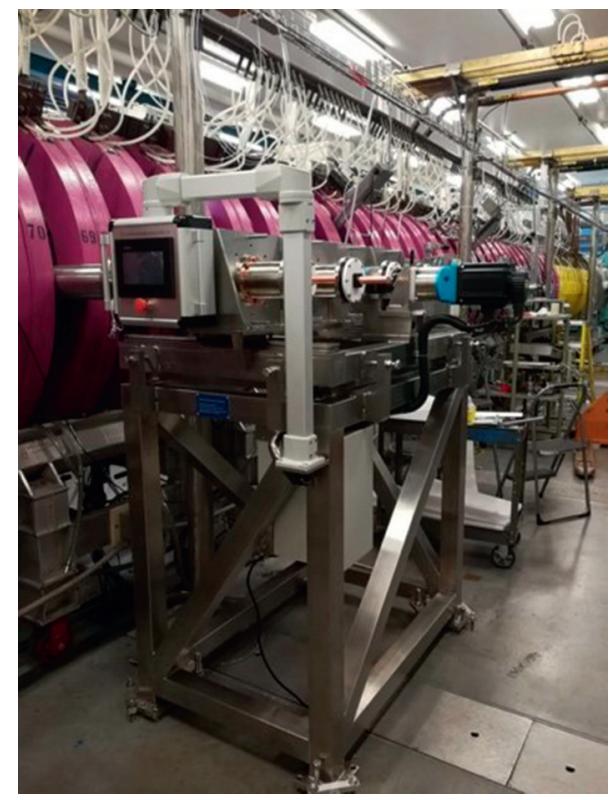

FIGURE 11: HHFW antenna integration with LAPD.

current on the antenna box and FS is reduced after optimization, whereas the maximum $J$ density decreases from $53.3 \mathrm{kA}$ to $14.5 \mathrm{kA}$. The reflection performance of the port at $30 \mathrm{MHz}$ is also improved after the structural optimization. The $k_{/ /}$spectrum distribution is sharper at the monopole phase $(0,0,0,0)$ and dipole phase $(0, \pi, 0, \pi)$ and $(0,90$, $270,180)$ than other phases. The optimized antenna can obtain a maximum $\left|\mathrm{k}_{/ /}\right|$spectrum, which peaks about $\left|\mathrm{k}_{/ /}\right|=$ $30 \mathrm{~m}^{-1}$ at the dipole phase $(0, \pi, 0, \pi)$. Furthermore, structure analysis of the antenna was carried out for optimization to meet the requirements of easy installation and steady and safe operation. In addition, the assembly strategy was carefully considered and formulated, which will help engineers to understand the assembly process and to install antennas in a highly efficient manner.

In summary, in collaboration with TAE, ORNL, and UCLA partners, the HHFW antenna mechanical design and fabrication have been completed. The design strategy and analysis results in this paper can provide useful reference and guidance for the study of HHFW antenna design and fabrication in LAPD or other magnetic confined fusion devices [17].

\section{Data Availability}

The figures used to support the findings of this study are available from TAE upon application at https://tae.com/.

\section{Conflicts of Interest}

The authors declare that they have no conflicts of interest.

\section{Acknowledgments}

This work was supported by the National Key R\&D Program of China under contract no. 2017YFE0300503.

\section{References}

[1] M. Alibakhshikenari, B. S. Virdee, M. Khalily et al., "Highgain on-chip antenna design on silicon layer with aperture excitation for terahertz applications," IEEE Antennas and Wireless Propagation Letters, vol. 19, no. 9, pp. 1576-1580, 2020.

[2] M. Alibakhshi-Kenari, M. Naser-Moghadasi, R. A. Sadeghzadeh, B. S. Virdee, and E. Limiti, "A new planar broadband antenna based on meandered line loops for portable wireless communication devices," Radio Science, vol. 51, no. 7, pp. 1109-1117, 2016. 
[3] M. Alibakhshikenari, B. S. Virdee, and E. Limiti, "Wideband planar array antenna based on SCRLH-TL for airborne synthetic aperture radar application," Journal of Electromagnetic Waves and Applications, vol. 32, no. 12, pp. 15861599, 2018.

[4] M. Alibakhshikenari, B. S. Virdee, C. H. See, R. A. Abd-Alhameed, F. Falcone, and E. Limiti, "High-isolation leaky-wave array antenna based on CRLH-metamaterial implemented on SIW with \pm 30 o frequency beam-scanning capability at millimetre-waves," Electronics, vol. 8, no. 6, p. 642, 2019.

[5] X. Yang, Y. Song, M. Binderbauer et al., "Overview of TAE technologies' HHFW project on LAPD," in Proceedings of the 6th International Conference on Biological Science (ICBS2019), Yogyakarta, Indonesia, October 2019.

[6] X. Yang, Y. Petrov, F. Ceccherini et al., "Simulations of high harmonic fast wave heating on the $\mathrm{C}-2 \mathrm{U}$ advanced beamdriven field-reversed configuration device," The European Physical Journal Conferences, vol. 157, p. 03065, 2017.

[7] S. J. Wukitch, M. L. Garrett, R. Ochoukov et al., "Characterization and performance of a field aligned ion cyclotron range of frequency antenna in Alcator C-Mod," Physics of Plasmas, vol. 20, no. 5, p. 056117, 2013.

[8] P. Jacquet, L. Colas, M.-L. Mayoral et al., "Heat loads on JET plasma facing components from ICRF and LH wave absorption in the SOL," Nuclear Fusion, vol. 51, no. 10, p. 103018, 2011.

[9] M. Bécoulet, L. Colas, S. Pécoul et al., "Edge plasma density convection during ion cyclotron resonance heating on Tore Supra," Physics of Plasmas, vol. 9, no. 6, pp. 2619-2632, 2002.

[10] R. J. Perkins, J. C. Hosea, G. J. Kramer et al., "High-harmonic fast-wave power flow along magnetic field lines in the scrapeoff layer of NSTX," Physical Review Letters, vol. 109, no. 4, p. 045001, 2012.

[11] F. W. Perkins, "Radiofrequency sheaths and impurity generation by ICRF antennas," Nuclear Fusion, vol. 29, no. 4, p. 583, 1989.

[12] R. Perkins, "Large RF field amplitudes in the SOL and far-field RF sheaths: a proposed mechanism for the anomalous loss of RF power to the SOL of NSTX," in Proceedings of the 26th IAEA Fusion Energy Conference, Kyoto, Japan, October 2016.

[13] D. A. D'ippolito, J. R. Myra, J. H. Rogers et al., "Analysis of RF sheath interactions in TFTR," Nuclear Fusion, vol. 38, no. 10, p. 1543, 1998.

[14] J. Tu, P. Song, and B. W. Reinisch, "Plasma sheath structures around a radio frequency antenna," Journal of Geophysical Research: Space Physics, vol. 113, no. 7, p. A07223, 2008.

[15] J. R. Wilson and P. T. Bonoli, "Progress on ion cyclotron range of frequencies heating physics and technology in support of the international tokamak experimental reactor," Physics of Plasmas, vol. 22, no. 2, p. 021801, 2015.

[16] W. Gekelman, P. Pribyl, Z. Lucky et al., "The upgraded large plasma device, a machine for studying frontier basic plasma physics," Review of Scientific Instruments, vol. 87, no. 2, p. 025105, 2016.

[17] G. A. Mesyats, D. I. Proskourovsky, and V. F. Puchkarev, "On effects resulting in appearance of new emission centers at explosive electron emission," in Proceedings of VIII International Symposium on Discharges and Electrical Insulation in Vacuum (ISDEIV), Albuquerque, NM, USA, 1978. 